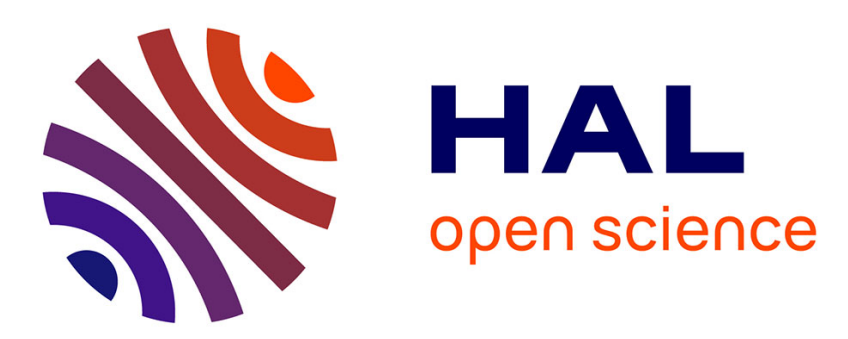

\title{
les enjeux sanitaires de la prise en compte de l'habitat mobile pour les gens du voyage \\ G Loiseau
}

\section{To cite this version:}

G Loiseau. les enjeux sanitaires de la prise en compte de l'habitat mobile pour les gens du voyage. Les inégalités de santé - Congrès national des observatoires régionaux de santé, Oct 2008, Marseille, France. hal-01784109

\section{HAL Id: hal-01784109 \\ https://hal-normandie-univ.archives-ouvertes.fr/hal-01784109}

Submitted on 3 May 2018

HAL is a multi-disciplinary open access archive for the deposit and dissemination of scientific research documents, whether they are published or not. The documents may come from teaching and research institutions in France or abroad, or from public or private research centers.
L'archive ouverte pluridisciplinaire HAL, est destinée au dépôt et à la diffusion de documents scientifiques de niveau recherche, publiés ou non, émanant des établissements d'enseignement et de recherche français ou étrangers, des laboratoires publics ou privés. 


\title{
D4 - Les enjeux sanitaires de la prise en compte de l'habitat mobile pour les gens du voyage
}

\author{
G. Loiseau ${ }^{a, b}$ \\ ${ }^{a}$ Département de sciences sociales, Université René-Descartes-Paris 5 , France ; ${ }^{b}$ Césam migrations \\ santé, Montpellier, France
}

\begin{abstract}
RESUME
Introduction. L'habitat mobile, lorsqu'il est utilisé comme résidence permanente, est entaché de deux représentations récurrentes. Tout d'abord, considéré d'un point de vue juridique comme étant « l'habitat traditionnel » des gens du voyage, il est largement culturalisé dans sa prise en compte. D'autre part, en tant qu'habitat non bâtit, il demeure perçu comme un habitat précaire et n'est pas considéré comme un logement. Au-delà d'une reconnaissance du mode de vie itinérant, la question du droit à l'habitat mobile mérite une attention particulière de la part des pouvoirs publics,
\end{abstract} d'autant plus qu'il représente un héritage culturel pour une partie de la population française.

Matériel et méthodes. A partir des observations recueillies dans le cadre d'une étude nationale commanditée par la Direction Générale de l'Action Sociale - sur l'accès aux droits sociaux des populations tsiganes en France, nous nous attacherons à montrer l'impact sanitaire de l'absence ou de la faible prise en compte de l'habitat mobile des gens du voyage. Nous compléterons ces données nationales par des éléments de terrain recueillis par l'association Césam migrations santé, assurant la médiation entre les collectivités locales et les gens du voyage dans l'Hérault.

Résultats. Les résultats de l'étude nationale font apparaître des difficultés récurrentes d'inscription de l'habitat mobile à l'échelon local, que cela soit dans le cadre d'une accession à la propriété par les gens du voyage ou d'un stationnement temporaire. S'ajoutent à cela des phénomènes de relégation qui se traduisent par une faible qualité environnementale (pollution sonore ou olfactive, toxicité du sol...) des sites d'installation des résidences mobiles. Qu'ils soient installés sur leurs propres terrains ou en itinérance, les gens du voyage rencontrent des difficultés permanentes à satisfaire leurs besoins vitaux, notamment l'accès à l'eau et à l'électricité. Ces difficultés génèrent des conditions de vie dégradées qui ont un impact sur la santé des personnes concernées (stress, maladies cardio-vasculaires, difficultés d'accès aux soins...).

Discussion et conclusion. Les gens du voyage sont conscients de vivre des «situations à risque » qui découlent majoritairement de l'inapplication des lois relatives à leur mode de vie. L'organisation et les comportements qu'ils adoptent eut-égard à leur habitat caravane permet d'affirmer que celui-ci n'est pas en tant que tel générateur d'inégalités de santé, mais que celles-ci sont bien plus à attribuer au traitement accordé, par les pouvoirs locaux, à cet habitat mobile et à la population qui y est associée. 


\section{1 - INTRODUCTION / OBJECTIFS}

Les « gens du voyage » se distinguent du reste de la population française par un mode d'habiter dont ils ont hérité et qui s'inscrit plus globalement dans un mode de vie fondé sur la dimension familiale. L'habitat mobile en question - qu'il soit ou non utilisé à des fins de mobilité géographique - dessert prioritairement, en ce qui concerne les Tsiganes, des logiques culturelles de vie en collectivités familiales élargies. Ainsi même pour des familles logées en dur, la caravane est souvent utilisée pour héberger les jeunes couples car elle permet de les maintenir au sein du foyer de vie.

La loi Besson du 5 juillet 2000 relative à l'accueil et à l'habitat des gens du voyage a franchi un pas dans la reconnaissance du mode de vie et d'habiter de ceux pour qui la «résidence mobile » constitue un « habitat traditionnel»; autrement dit les Tsiganes. Juridiquement la caravane est considérée avant tout comme une "habitation légère de loisirs », c'est-à-dire comme une résidence de seconde zone conçue pour n'être qu'occasionnellement substitutive à l'habitat «normé » qu'est l'habitat en dur. La loi Besson a permis une reconnaissance d'un mode de vie centré autour de l'habitat mobile en considérant que la caravane pouvait être « l'habitat permanent de leurs utilisateurs ». Elle introduit l'obligation pour les communes de prendre en compte cette forme d'habitat dans leurs documents d'urbanisme, au titre de la diversité de l'habitat. Néanmoins, elle alimente le phénomène d'ethnicisation qui est à l'œuvre dans le traitement social des gens du voyage en considérant qu'ils sont les seuls (au titre d'héritiers de cette forme d'habitat) à pouvoir bénéficier des dispositions spécifiques qu'elle prévoit. Or, la reconnaissance du mode d'habiter des gens du voyage trouve une de ses limites dans le risque pressenti par les pouvoirs publics, de voir I'habitat mobile se substituer au logement en dur pour des personnes qui y ont difficilement accès. Ainsi, aujourd'hui la difficulté première rencontrée par les gens du voyage ayant préservé un habitat mobile a trait à leur inscription (provisoire ou permanente) sur le territoire. Celle-ci dépend surtout de la possibilité de raccorder leur habitat caravane aux réseaux d'eau, d'électricité et d'assainissement qui conditionnent la décence du logement. Dans notre exposé nous évoquerons les conséquences de l'inapplication de la loi Besson sur la santé des personnes concernées.

\section{2 - MATERIELS / METHODES}

L'étude menée par le GEPECS (Paris 5) sous l'égide du ministère du travail, des relations sociales et de la solidarité et du ministère du logement et de la ville - Direction générale de l'action sociale - sur l'accès aux droits sociaux des populations tsiganes en France fait apparaître un certain nombre de discriminations à leur égard (Liégeois, 2007). Dans notre exposé, nous mettrons en corrélation la partie sur l'habitat et le logement et celle sur la santé. Nous décrirons également la réalité des conditions de vie des gens du voyage à partir des constats réalisés par l'association Césam migrations santé qui a en charge, depuis mai 2004, la médiation entre les gens du voyage et les collectivités locales sur le département de l'Hérault.

Les chercheurs de Paris 5 qui ont réalisé l'étude ont choisi six angles d'approche pour répertorier l'ensemble des difficultés d'accès aux droits sociaux pour ces populations : logement et l'habitat, droits du citoyen, droit à l'éducation, droits à l'initiative économique et à l'emploi, droits relatifs à la santé et aux prestations sociales, droits fondamentaux. Le choix du vocable « Tsiganes » dans la commande institutionnelle dénote une démarche novatrice consistant à identifier une population par son appartenance ethnique. Cette démarche a permis d'appréhender les difficultés rencontrées par les Tsiganes qui vivent en France, au-delà de la stricte entrée du mode de vie habituellement présentée sous la forme d'une graduation de leur mobilité (« sédentaires », « semi-sédentaires » et « itinérants »). En effet, cette vision est erronée dans la mesure où elle nie l'alternance entre des logiques d'ancrage et de mobilité géographique qui sont pourtant avérées chez ces populations.

L'étude s'est déroulée en trois temps: tout d'abord une analyse et une interprétation des textes législatifs et réglementaires concernant la thématique et la population ciblée. Ensuite, le recueil et l'analyse de la documentation existante (rapports publics, études diverses notamment issues du milieu associatif, travaux de recherche). Enfin, le recueil et l'analyse des données empiriques. A ce titre, des entretiens ont été réalisés, sur l'ensemble du territoire national, auprès des services de l'Etat et des collectivités locales, des organismes sociaux chargés de la prise en compte des Tsiganes et gens du voyage, des organismes chargés des questions relatives à la discrimination ou au racisme, de responsables associatifs tsiganes ou représentants des gens du voyage. Les 
chercheurs, tous spécialistes des populations tsiganes sur des sujets divers, ont également eu à charge de repérer un certain nombre de bonnes pratiques. Par ailleurs, les auteurs se sont attachés à présenter dans chacune des six parties, des situations précises illustrant les difficultés rencontrées par les Tsiganes en matière d'accès aux droits sociaux.

Rapidement les auteurs se sont rendu compte que la problématique de l'habitat était déterminante dans la prise en compte des gens du voyage et qu'elle avait des répercutions importantes dans beaucoup d'autres domaines.

Nous compléterons cette étude nationale par la présentation de situations récurrentes rencontrées par l'association Césam migrations santé (34) au cours de son travail de médiation entre gens du voyage et collectivités locales pour des situations de stationnement irrégulier. Les constats établis dans le cadre de ce travail de terrain viennent corroborer ceux établis par Lamia Missaoui (1999) concernant les trajectoires de santé des populations tsiganes ; à savoir que les problématiques de santé suscitent une mobilité géographique importante de la part de ces populations; et que l'agglomération montpelliéraine, équipée de structures médicales de grande renommée, attire une proportion importante de gens du voyage qui viennent s'y faire soigner ou faire soigner l'un des membres de leur famille.

Les observations relatives aux conditions de vie des gens du voyage venant séjourner dans l'Hérault pour des motifs médicaux ont été réalisées entre 2004 et 2005 dans un contexte de pénurie en matière d'accueil ( 80 places de caravanes pour des besoins estimés à 840 ), non résolu à ce jour.

\section{3 - RESULTATS}

La faible mobilisation des communes dans l'application de la loi Besson a une incidence directe sur leurs conditions de vie. Tout d'abord, cette situation génère un état de stress permanent pour les familles qui peuvent se faire expulser lorsqu'elles s'installent en dehors des aires d'accueil ; et qui subissent des pressions importantes de la part des élus, forces de l'ordre ou mêmes riverains, quand bien même les communes ne sont pas à jour de leurs obligations. Lorsqu'elles voyagent, les familles sont astreintes à un déplacement permanent et trouvent difficilement de possibilité de halte réunissant des critères de décence. Qu'elle soit régulière ou « sauvage », la halte s'effectue le plus souvent dans un environnement nocif pour la santé. Sur les aires d'accueil, des nuisances sonores importantes (proximité immédiates de voies de circulation ou de chemins de fer), olfactives (proximité d'usines) ou mêmes sanitaires (présence de rongeurs) ont été repérées de façon régulière. Trop souvent conçus par les élus dans l'unique but de s'affranchir des problèmes générés par l'installation inopinée des caravanes sur le territoire communal, ces équipements publics, très standardisés, révèlent des négligences importantes en ce qui concerne la qualité du cadre de vie (effet « parking » des emplacements, absence d'espaces verts ou d'aires de jeux pour les enfants, effet ghettoïsant induit par des clôtures hautes visant à camoufler les caravanes, etc.). Par ailleurs, les principes de gestion de ces lieux dévoilent des stratégies de contrôle social plus que d'accueil (relevé des plaques d'immatriculation, dépôts des cartes grises et parfois même présentation des carnets de vaccination des enfants) et portent parfois atteinte à la vie privée et familiale des résidents (interdiction d'accueillir des amis ou de la famille par exemple). Les gens du voyage se retrouvent aujourd'hui dans une situation d'assignation à résidence sur ces équipements publics dont le fonctionnement révèle souvent des mécanismes de relégation.

En dehors des aires d'accueil, les gens du voyage rencontrent des difficultés importantes pour accéder à l'eau et à l'électricité qu'ils soient en situation d'itinérance ou d'installation durable sur un terrain leur appartenant. Les demandes d'autorisation de raccordement aux fluides (eau et électricité) sont quasi-systématiquement refusées - quand bien même il s'agit de besoins vitaux et génèrent des situations de raccordements illégaux aux bornes incendie et aux réseaux d'électricité dans des conditions périlleuses que ce soit pour les protagonistes eux-mêmes ou pour autrui. Certaines familles parviennent à s'assurer une certaines autonomie (cuves d'une capacité de plusieurs centaines de litres installées dans les fourgons, groupes électrogènes pour l'alimentation électrique). Mais pour les plus précaires, qui dépendent complètement des réseaux publics, ces situations d'exclusions se traduisent par des difficultés importantes en ce qui concerne la possibilité de chauffage l'hiver, de climatisation des caravanes l'été ou encore d'hydratation des personnes fragiles. Ces situations sont aggravées lorsqu'il y a des malades dont le traitement 
nécessite une immobilisation physique ou bien, comme nous l'avons observé à plusieurs reprises, un raccordement à l'électricité (personnes sous respirateur artificiel). II apparaît également que la parole des gens du voyage est régulièrement mise en doute par les communes lorsqu'ils justifient par un problème médical leur nécessité d'obtenir une autorisation de stationnement ou un accès aux fluides, y compris sur leur propre terrain.

Les gens du voyage établissent généralement la corrélation entre leur environnement de vie immédiat et leurs problèmes de santé. L'étude nationale fait apparaître qu'il n'existe pas de « pathologies tsiganes » mais des pathologies qui témoignent de la précarité des conditions de vie (importance des maladies cardio-vasculaires par exemple). Les gens du voyage déploient une forte mobilisation, dans la mobilité, en cas de problème de santé d'un des leurs. Etant nombreux à venir accompagner et soutenir le malade, il leur est souvent difficile de s'installer sur les aires d'accueil. II n'est donc pas rare qu'ils séjournent dans des conditions plus qu'aléatoires alors qu'ils ont parmi eux un ou plusieurs malades.

Les pouvoirs publics se sont penchés sur la question de la santé des gens du voyage notamment dans le cadre de programmes régionaux. Une prise en compte sanitaire de cette population a été initiée dans près de la moitié des programmes régionaux d'accès à la santé et aux soins (PRAPS), avec une approche se voulant le plus souvent culturaliste (actions de prévention par rapport aux pratiques alimentaires ou actions de médiation pour favoriser les relations entre gens du voyage et personnel médical). II existe donc une inadéquation entre les actions proposées et les raisons évoquées pour expliquer l'état de santé de ces populations. Ce décalage témoigne d'une difficulté pour les institutions sociales et sanitaires à intervenir directement pour l'amélioration immédiate des conditions de vie des gens du voyage, qui restent soumises à la volonté des pouvoirs locaux y compris lorsqu'ils sont installés sur des terrains dont ils sont propriétaires.

\section{4 - DISCUSSION / CONCLUSION}

Les droits des gens du voyage, citoyens français inscrits depuis plusieurs siècles sur le territoire national, sont régulièrement bafoués au nom de la marginalité qu'ils incarnent du fait de leur habitat mobile, jugé précaire. Le mode de vie des gens du voyage met en jeu, en exergue et en contraste deux principes fondamentaux de notre société que sont la liberté de circulation et le droit de propriété.

Les personnes qui naissent et grandissent en France dans un habitat mobile sont désavantagées en matière de santé par rapport au reste de la population française. Cette inégalité n'est pas à associer à une hypothétique précarité de l'habitat mobile, mais bien au traitement qui lui est accordé par les pouvoirs publics. Par exemple, aucun texte législatif ne permet aujourd'hui aux gens du voyage d'opposer une problématique médicale pour l'obtention d'autorisations de stationnement. Quelques villes ont solutionné ce problème en créant (parfois au sein même des hôpitaux) des emplacements réservés aux gens du voyage ou en créant des aires d'accueil à proximité immédiate des hôpitaux.

Les inégalités de santé que connaissent les gens du voyage sont liées aux conditions de vie qui leur sont faites et qui sont la conséquence directe de l'inapplication de la loi Besson. Les pouvoirs publics ne retiennent le plus souvent qu'un aspect restrictif de cette loi, à savoir l'obligation d'accueil pour les communes présentée comme solution aux problèmes de stationnement illégal (autorisant en contrepartie l'expulsion des groupes s'installant en dehors de ces équipements). Or, si les aires d'accueil sont une réponse adaptée aux difficultés de stationnement, elles ne répondent pas à la dimension de l'ancrage territorial que revendiquent pourtant une proportion importante des familles concernées. Une prise en compte plus globale de l'habitat mobile à l'échelle territoriale permettrait une amélioration des conditions de vie de cette population.

Le refus des pouvoirs publics de considérer l'habitat caravane comme un logement (empêchant l'octroi des allocations logement ou le recours à des prêts bancaires préférentiels lors de son achat) traduit une conception de cet habitat en terme de «choix de vie ». Or cette dimension de « choix », souvent alléguée par les élus, n'est que très succincte, voire inexistante pour les gens du voyage qui grandissent et apprennent à vivre en caravane. Elle existe par contre pour d'autres catégories de populations, non issues du «monde du voyage », qui pour des raisons diverses (économiques, idéologiques, sociales...) font le choix de vivre en habitat mobile (caravane, mobile 
home, etc.) et subissent les mêmes préjudices que les gens du voyage sans pour autant bénéficier des dispositifs conçus pour eux. Ainsi, la circulaire du 17 décembre 2003 « relative aux terrains familiaux permettant l'installation des caravanes constituant l'habitat permanent de leurs utilisateurs » permet de financer des projets d'aménagements privés ou même locatifs sur des budgets d'Etat prévus pour la réalisation des aires d'accueil ; mais concerne exclusivement les personnes identifiées comme gens du voyage. Or, les personnes qui font le choix de vivre en habitat mobile sans en être héritiers ne se reconnaissent pas dans cette catégorie qui, bien qu'ayant été forgée par l'administration, demeure fortement ethnicisée. L'apparition de ces nouvelles populations inscrites dans des schémas d'habitat similaires à ceux habituellement adoptés par les gens du voyage génère des réflexions notamment concernant la nécessité d'étudier et de proposer des critères de décence du logement appliqués à l'habitat mobile. A ce sujet, les gens du voyage adoptent généralement des comportements exemplaires en ce qui concerne l'entretien de leur caravane et la prévention des risques liés à la fragilité de leur habitat, dont ils savent qu'il n'est pas conçu « pour durer ».

\section{REFERENCES}

Loiseau, G (2007). « L'accès aux droits relatifs à la santé et aux prestations sociales », in Liégeois, JP. L'accès aux droits sociaux des populations tsiganes en France. Rennes : Editions ENSP, p. 153-193.

Missaoui L. (1999), Gitans et santé de Barcelone à Turin. Les compétences de l'étranger de l'intérieur: ethnicité et métissages chez les gitans catalans et andalous autour des problèmes de santé publique, Canet : Llibres del Trabucaïre.

Robert, C (2007). «L'accès au logement et à l'habitat », in Liégeois, JP. L'accès aux droits sociaux des populations tsiganes en France. Rennes : Editions ENSP, p. 37-69. 